\title{
LASER SCAN REGISTRATION USING PLANAR FEATURES
}

\author{
M. Previtali ${ }^{\text {a }}$, L. Barazzetti ${ }^{\text {a }}$, R. Brumana ${ }^{\text {a }}$, M. Scaioni ${ }^{\text {b }}$ \\ ${ }^{\text {a }}$ Politecnico di Milano, Department of Architecture, Built Environment and Construction Engineering \\ via G. Ponzio 31, Milano, Italy - emails: [mattia.previtali, luigi.barazzetti, raffaella.brumana]@polimi.it \\ ${ }^{\mathrm{b}}$ Tongji University, College of Surveying and Geo-Informatics \\ 1239 Siping Road, 200092 Shanghai, P.R. China - email: marco@tongji.edu.cn
}

Commission V, WG 3

KEY WORDS: Registration, Laser scanning, Point cloud, Segmentation

\begin{abstract}
:
Point cloud acquisition by using laser scanners provides an efficient way for 3D as-built modelling of indoor/outdoor urban environments. In the case of large structures, multiple scans may be required to cover the entire scene and registration is needed to merge them together. In general, the identification of corresponding geometric features among a series of scans can be used to compute the 3D rigid-body transformation useful for the registration of each scan into the reference system of the final point cloud. Different automatic or semi-automatic methods have been developed to this purpose. Several solutions based on artificial targets are available, which however may not be suitable in any situations. Methods based on surface matching (like ICP and LS3D) can be applied if the scans to align have a proper geometry and surface texture. In the case of urban and architectural scenes that present the prevalence of a few basic geometric shapes ('Legoland' scenes) the availability of many planar features is exploited here for registration. The presented technique does not require artificial targets to be added to the scanned scene. In addition, unlike other surface-based techniques (like ICP) the planar feature-based registration technique is not limited to work in a pairwise manner but it can handle the simultaneous alignment of multiple scans. Finally, some applications are presented and discussed to show how this technique can achieve accuracy comparable to a consolidated registration method.
\end{abstract}

\section{INTRODUCTION}

In the last years a great attention was paid to the development of terrestrial laser scanning (TLS) applications in different domains. This is mainly due to the chance to acquire both large surfaces and fine details in automatic way.

A great attention has been recently paid to reconstruction of asbuilt building models starting from TLS point clouds, not only in the field of cultural heritage preservation, where surfaces are usually complex and irregular, but also for large and medium size civil structures. In addition, an important transition to Building Information Modelling (BIM) is taking place in the Architecture, Engineering, and Construction domain (AEC Azhar, 2011). Indeed, in a BIM model not only the geometric aspects are considered, but also semantic and additional information can be included to have a deeper understanding of the existing buildings. Thus, both interiors and external façades of a building should be properly surveyed.

Due to the large extents of the objects, multiple scans are often needed and their registration is a mandatory task. Since urban environments mainly consist of planar objects, a registration method based on the identification of corresponding planar features between the acquired point clouds can be effectively used as an alternative to standard registration procedures (see a review in Subsect. 1.1).

This paper presents a new registration method for the registration of scans including predominantly planar features, like those captured in indoor building environments. As shown in next subsection, the possibility of using geometric features for scan registration is a well know topic in the literature and similar approaches already exist. For this reason the scan registration methodology discussed in this paper (see Sect. 2) is not revolutionary, but it simply attempts to increase the automation degree and the robustness of previous implementations. A new segmentation algorithm is the core of scan registration technique (Sect. 3). In addition, a comparison between the results obtained from the presented methodology and those coming from a consolidated solution are presented in Section 5 .

\subsection{Brief analysis of automatic registration techniques}

Extensive research for developing automatic registration procedures for laser scans has been carried out. Only a brief analysis is reported here for the purpose of introducing the method addressed in this paper. For a more comprehensive discussion and overview on the literature on this topic, the reader is referred to Vosselman and Maas (2010) and Barnea and Filin (2010).

Target-based approaches are the most commonly employed and implemented techniques in commercial software packages. This solution is also quite popular among practitioners. Artificial targets are added to the scanning scene and used as corresponding elements to estimate a rigid-body transformation between two scans (Scaioni, 2012). Targets are made of special highly-reflective materials and/or with a particular shape that can be automatically detected and matched in any scans. However, artificial elements have to be added to the scene, which is not always feasible or economically convenient, for example in the case of cultural heritage sites, impervious rock cliffs, or large settlements. Target-based registration methods usually work in pairwise manner, but also multi-scan approaches exist (see, e.g., Scaioni and Forlani, 2003). In addition, targets may introduce systematic errors that need to be properly modelled in high-precision applications (Alba et al., 2008).

In many applications, surface matching techniques are used for scan registration. Among all these methods, the Iterative Closest Point (ICP) algorithm (Besl and McKay, 1992) and its improvements (see Rusinkiewicz and Levoy, 2001) is the most exploited algorithm. ICP for point cloud registration works without any pre-knowledge about the point-to-point correspondences, except a preliminary rough pre-alignment which can be carried out by manual identification of at least 
three corresponding features in a pair of scans. ICP establishes point-to-point correspondence iteratively based on the minimum Euclidean distance. If the initial values are good enough, this procedure usually quickly converges. ICP is a pairwise registration procedure and cannot reliably handle simultaneous registration of multiple scans. This results in the propagation of registration errors when more scans are acquired and added to a project. A multi-scan ICP solution was proposed by Williams and Bennamoun (2001), but in the knowledge of the authors it did not follow up into regular practice.

Another surface-based approach is the Least Squares 3D Surface Matching (LS3D) method proposed by Gruen and Akca (2005). LS3D estimates the transformation parameters of one or more 3D search surfaces with respect to a template. It can be considered as an extension of the Least Squares Matching (Gruen, 1985) algorithm developed for measuring corresponding points in images. In LS3D the minimization is carried out using the sum of squares of the Euclidean distances between the surfaces. Also a solution incorporating laser intensity values in the registration process was proposed in Acka (2007).

Other registration techniques exist that are generally applied only for scientific purpose, even though their potential is remarkable. For example, laser intensity-based techniques (Böhm and Becker, 2007; Wang and Brenner, 2008; Kang et al., 2009) fall into this category. Some corresponding features are detected in automatic way in the intensity images that are associated to points in a 3D scan. These points are then used as in standard target-based approaches to estimate a 3D rigid body transformation. The main advantage of this technique is that no artificial targets are required. However, a good texture and a large overlap are generally required to provide a sufficient number of reliable correspondences (Alba et al., 2011).

In Al-Manasir and Fraser (2006) a camera mounted on top of the laser scanner is used for scan registration. Indeed, the relative orientation between images taken from different stations provides the transformation when the relationship between camera and laser coordinate systems is known. Even though the use of RGB images for scan registration might be useful in some applications, some laser scanners do not have an integrated digital camera. However, additional camera stations are required to strengthen the network geometry adopted to compute the registration parameters of each scan station, as proposed in Moussa and Fritsch (2014). In addition, mapping between imagery and point cloud might suffer from coregistration errors and from different spatial resolutions of both datasets.

In recent years, the availability of laser scanners equipped with tools enabling the direct georeferencing has increased the interest towards this technique (Lichti and Gordon, 2004; Scaioni, 2005). Generally speaking, a laser scanner becomes a sort of theodolite: it can be mounted on a tripod, and then the instrument is setup in horizontal position using a bubble level or an automatic inclinometre. The orientation in the horizontal plane can be estimated with a telescope or a reference target. The alignment of multiple scans is accomplished using the traversing technique.

Finally, geometric feature-based registration techniques are presented in the literature (e.g., Dold and Brenner, 2006; Rabbani et al., 2007; Wang and Brenner, 2008; and Van Goor et al., 2011). Indeed, man-made and urban environments mainly consist of objects that can be modelled by using a set of a few well defined geometric shapes. Determining the transformation parameters of different scans can therefore be based on geometric features present in the scene (Dijkman and van den Heuvel 2000). Compared to standard surface registration approaches (like ICP), registration with features allows for global registration.

It deserves to be mentioned that the integration between point clouds at non-homogenous resolution is sometimes required, for example when laser scans acquired from different platforms or techniques (e.g., laser scanning and photogrammetry) have to be merged. Special solutions have been developed to this purpose (see, e.g., Novak and Schindler, 2013).

\section{SCAN REGISTRATION OVERVIEW}

The aim of this research was to develop a registration method for laser scans gathered inside a building room for the purpose of modelling the indoor geometry. After exclusion of using targets which require additional workload for their deployment, the decision fell on the exploitation of the main property of building indoor rooms, i.e., the prevalence of planar features. Also the adoption of ICP-like methods was discarded because the poor control on the quality of registration. Indeed, as noted by Bennamoun and Mamic (2002) ICP just produces registration without giving any information about reliability and confidence of the estimated registration parameters. Conversely the presented approach is a direct application of Least Squares (LS) fitting and gives a full covariance matrix of the estimated parameters.

The developed scan registration procedure based on planar features (see workflow in Fig. 1) works out the solution in the following four steps:

1. points are labelled as belonging to a certain planar object. This is done for several objects in different unregistered scans. This step can be automated by using the segmentation algorithm presented in Section 3 ;

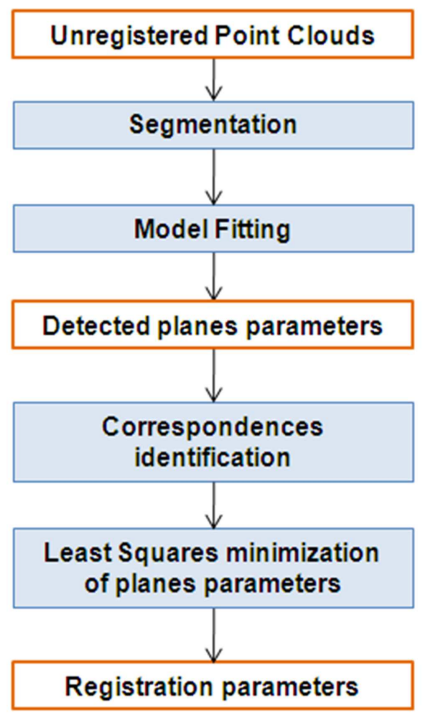

Figure 1. Workflow of the developed method for scan registration with planar features.

2. a LS fitting algorithm calculates the object parameters for every object in each scan;

3. correspondences between planes belonging to different scans are established using exhaustive search in the parameter space. In particular, the process operates between a couple of scans identifying a 
minimal set of correspondences. In the case of planes, a minimum of three correspondences are required. This minimum set is used to demine an initial approximated registration values between the scan pair which are then used to evaluate correspondences between all other planes. Between all the computed solutions the one maximizing the number of correspondences is used; and

4. the final parameters of 3D rigid-body transformation are estimated by LS.

In the next sections the developed segmentation procedure and the LS fitting of plane parameters are chiefly focused, since the other steps of the developed approach are quite standard tasks.

\section{PLANAR FEATURE SEGMENTATION}

A first step towards scan registration is the identification of all planar features in the scene. Detection of planar objects is accomplished by using a specifically modified RANSAC algorithm (Fischler and Bolles, 1981). Workflow is shown in Figure 2.

This implementation is aimed at reducing spurious results obtained by the standard sequential RANSAC segmentation as reported by several works in the literature (Boulaassal et al., 2008; Awwad et al., 2010). Those bad-segmentation problems can be categorized into under- and over-segmentation. Undersegmentation is generally due to the fact that points resulting in the maximum consensus to RANSAC may belong to different objects. Over-segmentation is generally associated with noise or irregularities in the data set that are not evaluated in the RANSAC estimation of inliers. This may result in a wrong subdivision of a single element into several objects.

To partially overcome the previous enlisted limitations, a new automatic approach is presented for the segmentation of planar surfaces based on the combination of RANSAC and regiongrowing techniques (Sapkota, 2008). The aim of this strategy is to derive 'meaningful' segments from building point clouds. This means that extracted segments would correspond to semantic objects of interest (e.g., floors, walls, roofs, etc.) instead of selecting those which simply best fit some blind mathematical models. This hybrid strategy allows combining the robustness of RANSAC with the spatial proximity used in region growing methods. Indeed, in contrast to previously published methods (Tóvári and Pfeifer 2005; Rabbani, 2006) the segmentation results are not dependent on the selection of 'seed' points because the estimation of planar segment is performed by using RANSAC. In addition fewer parameters are required with respect to the region-growing implementation presented in Vosselman et al. (2004), where the selection of slightly different values of control parameters may result in a large variety of bad-segmentation problems. On the other hand, the developed segmentation procedure that incorporates topology information is able to solve for the critical situations reported in Boulaassal et al. (2009), for instance.

In particular, under-segmentation is reduced introducing information about point topology. Indeed, even if points are not usually related by any topological relationship in a point cloud, we can assume that points belonging to the same object should be sufficiently close to one another, while groups of points belonging to different objects should be interspaced. For this reason, point cloud proximity is evaluated by using a 2D binary occupancy raster map (BORM) where occupied regions are assigned value 1 . To work this out, points belonging to the same plane are projected orthogonally onto a bitmap. All pixels in the bitmap containing at least one projected point are assigned value 1 , while others value 0 . This raster map allows finding connected regions of pixels featuring value 1 . Then all points whose projection belongs to the same connected component are clustered.

Once all planar elements are detected, the extracted planes are clustered together to reduce over-segmentation problems. Object clustering is performed by evaluating three parameters: (i) similarity of normal vectors; (ii) perpendicular distance between planes; and (iii) intersection between clusters.

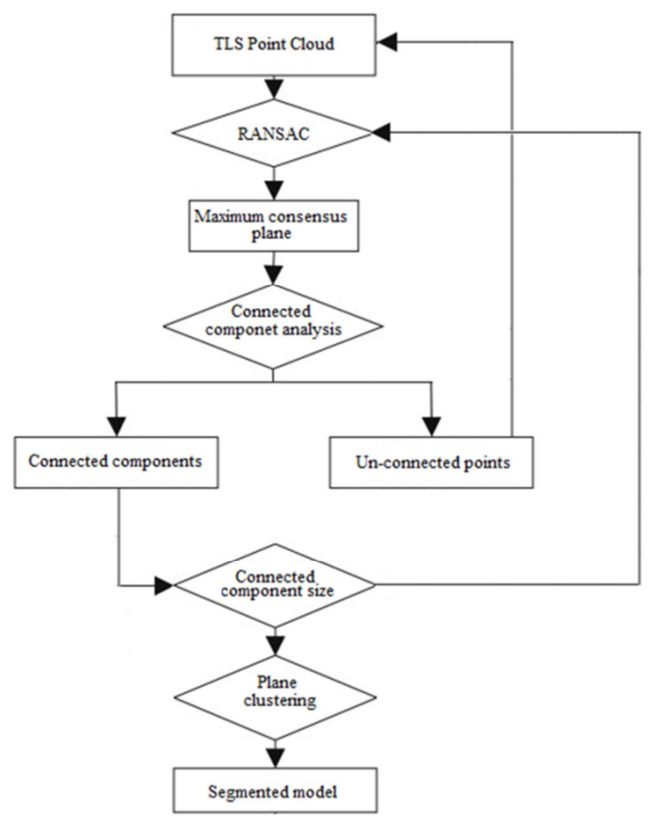

Figure 2. Workflow of the developed segmentation process for planar features.

\section{SCAN REGISTRATION}

\subsection{Formulation of the registration problem}

The registration problem between two scans is usually formulated by assuming one as reference (or 'master') and the other as a 'slave' to be mapped on it. Six parameters casted in the spatial rotation matrix $\mathbf{R}$ and the $3 \mathrm{D}$ shift vector are used to define a rigid-body transformation to map features (for example, points or planar features) from 'slave' $\left(\left\{\mathrm{F}_{s}\right\}\right)$ to 'master' $\left(\left\{\mathrm{F}_{m}\right\}\right)$ :

$$
\left\{\mathrm{F}_{\mathrm{m}}\right\}=\mathbf{R}\left\{\mathrm{F}_{s}\right\}+T
$$

In the case more than two scans have to be registered together, a block approach may be used to entail also features which are visible on multiple scans (i.e., in more than two). In this case a different formulation of the registration problem is needed, keeping into account that a global reference system (GRS) has to be introduced. Such GRS may be the reference system of a single scan, as in the previous case, or may be defined by introducing external (ground control points) or inner constraints (Dermanis, 1994).

The equation adopted to describe the 3D rigid-body transformation becomes:

$$
\left\{\mathrm{F}_{G R S}\right\}=\mathbf{R}_{i}\left\{\mathrm{~F}_{i}\right\}+T_{i}
$$


where $\left\{\mathrm{F}_{G R S}\right\}$ are the estimated features in the GRS, $\left\{\mathrm{F}_{i}\right\}$ are the observed features in the $i$-th scan, and $\mathbf{R}_{i}$ and $\mathrm{T}_{i}$ define the 3D rigid-body transformation from the reference system of the $i$-th scan to GRS. Several Eq.s (2) can be incorporated into a system of equations including also pseudo-observations to setup the datum definition

\subsection{Implementation with planar features}

As previously discussed, in urban environments many objects consist of one or more planar faces, which can be used for registration. Describing a plane by the normal vector $n=\left[\mathrm{n}_{\mathrm{x}} \mathrm{n}_{\mathrm{y}}\right.$ $\left.\mathrm{n}_{\mathrm{z}}\right]^{\mathrm{T}}$ and the perpendicular distance from the origin $\rho$ (Fig. 3) provide a singularity free representation for infinite planes (Van den Heuvel, 1999). This representation is also known as Hesse form of the plane and is more suitable for the LS solution than other parameterizations.

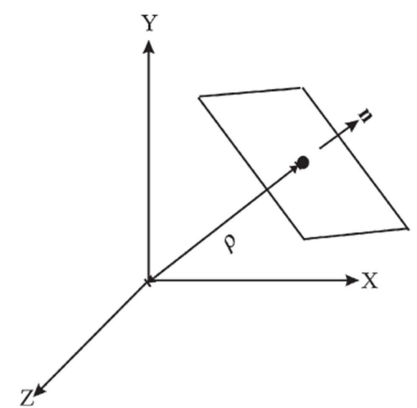

Figure 3. Parameters of the plane in the parameterization proposed in Van den Heuvel (1999): normal $n$ and distance from the origin $\rho$.

Once a plane is detected by means of the segmentation strategy presented in the previous Section, its equation is estimated by means of LS fitting. In particular, given a general plane in the form:

$$
a x+b y+c z+d=0
$$

the normal vector $n=\left[\mathrm{n}_{\mathrm{x}} \mathrm{n}_{\mathrm{y}} \mathrm{n}_{\mathrm{z}}\right]^{\mathrm{T}}$ is simply determined observing that:

$$
\begin{aligned}
& \mathrm{n}_{\mathrm{x}}=\frac{\mathrm{a}}{\sqrt{\mathrm{a}^{2}+\mathrm{b}^{2}+\mathrm{c}^{2}}} \\
& \mathrm{n}_{\mathrm{y}}=\frac{\mathrm{b}}{\sqrt{\mathrm{a}^{2}+\mathrm{b}^{2}+\mathrm{c}^{2}}} \\
& \mathrm{n}_{\mathrm{z}}=\frac{\mathrm{c}}{\sqrt{\mathrm{a}^{2}+\mathrm{b}^{2}+\mathrm{c}^{2}}}
\end{aligned}
$$

As a plane has only three degrees of freedom, a constraint on the length of normal vector $n$ is introduced:

$$
\|n\|=\sqrt{\mathrm{n}_{\mathrm{x}}^{2}+\mathrm{n}_{\mathrm{y}}^{2}+\mathrm{n}_{\mathrm{z}}^{2}}=1
$$

while the distance of the plane from the origin $\rho$ is (Gellert et al., 1989):

$$
\rho=\frac{d}{\sqrt{a^{2}+b^{2}+c^{2}}}
$$

The estimation of registration parameters is obtained by LS minimization of the sum of squared differences. Mathematically it can be expressed as follows:

$$
\min _{\{\mathbf{R}\},\{T\}} \sum_{i=1}^{C}\left[\Psi_{\mathbf{R}, T}\left(n_{i, 1}, \rho_{i, 1}\right)-\Psi_{\mathbf{R}, T}\left(n_{i, 2}, \rho_{i, 2}\right)\right]^{2}
$$

where there are $\mathrm{C}$ correspondences between planes belonging to different scans. Each correspondence is established between two objects 1 and 2. $\Psi_{\mathrm{R}, \mathrm{T}}$ is an operator that applies the transformation, defined by $\mathbf{R}$ and $T$, to the plane parameters $n_{\mathrm{i}, \mathrm{k}}$ and $\rho_{\mathrm{i}, \mathrm{k}}(\mathrm{h}=1,2)$. In the following, the case of co-registration of two scans is proposed, based on Eq. (1) model. In a similar way is also possible to deal with multiple scans, using the model in Eq. (2).

Each plane provides three equations for the difference along the components of the normal vector $n_{\delta}$ and one equation for the difference along the distance from the origin $\rho_{\delta}$ :

$$
\begin{gathered}
n_{\delta}=n_{1}-\left(\mathbf{R} \cdot n_{2}\right) \\
\rho_{\delta}=\rho_{2}-\rho_{1}+\left(\mathbf{R} \cdot n_{2}\right) \cdot T
\end{gathered}
$$

From Eq. (8) it is possible to observe that the normal is only affected by rotation, whereas Eq. (9) shows that the change in $\rho$ is a function of both translation and rotation of the scan. To solve the non-linear LS problem in Eq. (7) the Gauss-Newton method is used after linearization. In particular, by parameterizing $\mathbf{R}$ with Euler angles $\Omega, \Phi$ and K, a system of Eqs. (8) and (9) can be linearized as follows:

$$
\mathbf{A} \cdot \Delta x=\delta+v
$$

where the design matrix $\mathbf{A}$ is:

$$
\mathbf{A}=\left[\begin{array}{cccccc}
\frac{\partial n_{\delta, x}}{\partial \Omega} & \frac{\partial \rho_{\delta, x}}{\partial \Phi} & \frac{\partial \rho_{\delta, x}}{\partial \mathrm{K}} & 0 & 0 & 0 \\
\frac{\partial n_{\delta, y}}{\partial \Omega} & \frac{\partial \rho_{\delta, y}}{\partial \Phi} & \frac{\partial \rho_{\delta, y}}{\partial \mathrm{K}} & 0 & 0 & 0 \\
\frac{\partial n_{\delta, z}}{\partial \Omega} & \frac{\partial \rho_{\delta, z}}{\partial \Phi} & \frac{\partial \rho_{\delta, z}}{\partial \mathrm{K}} & 0 & 0 & 0 \\
\frac{\partial \rho_{\delta}}{\partial \Omega} & \frac{\partial \rho_{\delta}}{\partial \Phi} & \frac{\partial \rho_{\delta}}{\partial \mathrm{K}} & \frac{\partial \rho_{\delta}}{\partial T_{x}} & \frac{\partial \rho_{\delta}}{\partial T_{y}} & \frac{\partial \rho_{\delta}}{\partial T_{z}}
\end{array}\right]
$$

The partial derivatives of Eqs. (8) and (9) with respect to the registration parameters are evaluated with respect to the actual approximate values. $\Delta x$ is the vector of unknown corrections to the approximate values of the registration parameters:

$$
\Delta x=\left[\begin{array}{llllll}
d \Omega & d \Phi & d \mathrm{~K} & d T_{x} & d T_{y} & d T_{z}
\end{array}\right]^{\mathrm{T}}
$$

Vector $\delta$ contains differences between measured and computed plane parameters on the basis of approximate values:

$$
\delta=\left[\begin{array}{c}
n_{1, x}-\left(\mathbf{R} \cdot n_{2, x}\right) \\
n_{1, y}-\left(\mathbf{R} \cdot n_{2, y}\right) \\
n_{1, z}-\left(\mathbf{R} \cdot n_{2, z}\right) \\
\rho_{2}-\rho_{1}+\left(\mathbf{R} \cdot n_{2}\right) \cdot T
\end{array}\right]
$$

Vector $v$ contains residuals. 
By parameterizing $\mathbf{R}$ with Euler angles, the partial derivatives with respect to rotation are as follows:

$$
\begin{gathered}
\frac{\partial n_{\delta}}{\partial \Omega}=-\frac{\partial \mathbf{R}}{\partial \Omega} n_{2} ; \frac{\partial n_{\delta}}{\partial \Phi}=-\frac{\partial \mathbf{R}}{\partial \Phi} n_{2} ; \frac{\partial n_{\delta}}{\partial \mathrm{K}}=-\frac{\partial \mathbf{R}}{\partial \mathrm{K}} n_{2} \\
\frac{\partial \rho_{\delta}}{\partial \Omega}=\left(\frac{\partial \mathbf{R}}{\partial \Omega} n_{2}\right) \cdot T ; \frac{\partial \rho_{\delta}}{\partial \Phi}=\left(\frac{\partial \mathbf{R}}{\partial \Phi} n_{2}\right) \cdot T ; \frac{\partial \rho_{\delta}}{\partial \mathrm{K}}=\left(\frac{\partial \mathbf{R}}{\partial \mathrm{K}} n_{2}\right) \cdot T
\end{gathered}
$$

For translation vector $T$ the partial derivatives are:

$$
\begin{gathered}
\frac{\partial n_{\delta}}{\partial \mathrm{T}_{\mathrm{i}}}=0 \\
\frac{\partial \rho_{\delta}}{\partial \mathrm{T}_{\mathrm{i}}}=\left(\mathbf{R} \cdot n_{2}\right) \cdot \frac{\partial T}{\partial \mathrm{T}_{\mathrm{i}}}
\end{gathered}
$$

As can be seen from Eqs. (8) and (9), planes contribute to $T$ only for the direction of the normal vector $n$. For example, a plane which is parallel to the $\mathrm{X}$ axis will contribute only in the estimation of $T_{x}$, while no contribution will be given in the estimation of $\mathrm{T}_{\mathrm{y}}$ and $\mathrm{T}_{\mathrm{z}}$. For this reason, planes should be evenly distributed on the scene in order to allow a proper estimation of $T$. A good environment for the presented scan registration strategy is represented by indoor scans. Indeed, in such case room's walls, floor and ceiling give a robust plane configuration for registration purpose.

The proposed cost function presumes equal weighting and uncorrelated object parameters. These assumptions may not be appropriate since significant differences in parameter precision and large correlations between parameters may exist, depending upon data coverage of the object. These correlations can have negative effects on the convergence of this procedure. This problem can be tackled by weighting the equations using the inverse of the covariance matrix obtained during plane fitting.

\section{APPLICATIONS AND ACCURACY EVALUATION}

The procedure for scan registration through the use of planar features was applied to building indoor data sets having different characteristics in terms of network geometry and point density. Two examples are illustrated here to present the main advantages and disadvantages of this method in real case studies.

\section{1 'Classroom' data set}

The first example consists in the registration of a single scan pair. These data were acquired with a FARO-FOCUS 3D laser scanner (www.faro.com), which is based on phase-shift principle for range measurement. Some technical specifications are reported in Table 1.

The scene is a university classroom, whose dimensions are 8 $\mathrm{m} \times 4.5 \mathrm{~m} \times 3 \mathrm{~m}$. Each scan (Fig. $4 \mathrm{a}$ ) consists of 28 million points resulting in average point density of about $1 \mathrm{pt} / 3 \mathrm{~mm}$. The scans were segmented and planes recovered using the approach presented in Section 3. In particular, the parameters used to segment the two scans are presented in Table 2. The same parameters are used for both scans to have a uniform accuracy in the estimation of planes.

The number of recovered planes in Scans 1 and 2 are 15 and 16, respectfully. Among these, 14 were matched. The coordinate system of Scan 1 was defined as the GRS. The final sigma naught $\left(\sigma_{0}\right)$ of $L S$ solution was $3.0 \mathrm{~mm}$. The theoretical accuracy of transformation parameters obtained from the estimated covariance matrix is reported in Table 3 . Some relevant steps of the registration procedure are graphically shown in Figure 4.

To register the same data set, artificial targets were used and results compared to the ones obtained from the developed registration method. By using target-based alignment, a sigma naught of $2.0 \mathrm{~mm}$ was obtained. The difference between the translation vectors and the Euler angles obtained from both registration techniques directly provides information about the misalignment (Tab. 3). A further check was performed on the coordinates of the artificial targets measured in both projects, showing a mean difference of $2.1 \mathrm{~mm}$ and a standard deviation of $0.9 \mathrm{~mm}$.

\begin{tabular}{|c|c|}
\hline Range measurement mode & Phase-shift \\
\hline Operational range & $0.6-150 \mathrm{~m}$ \\
\hline Angular resolution & $0.009^{\circ}$ \\
\hline Measurement speed & $120.000-976.000$ points $/ \mathrm{sec}$ \\
\hline Precision at $\mathbf{1 0} \mathbf{~ m}$ & $0.6 \mathrm{~mm}(90 \%$ reflectivity $)$ \\
& $1.2 \mathrm{~mm}(10 \%$ reflectivity $)$ \\
\hline \multirow{2}{*}{ Precision at $\mathbf{2 5} \mathbf{~ m}$} & $0.95 \mathrm{~mm}(90 \%$ reflectivity $)$ \\
\hline
\end{tabular}

Table 1. Technical specifications of the adopted FARO-FOCUS 3D laser scanner.

\begin{tabular}{|c|c|}
\hline RANSAC plane threshold $\varepsilon$ & $1 \mathrm{~cm}$ \\
\hline RANSAC normal threshold $\alpha$ & $10^{\circ}$ \\
\hline Bitmap cell size $\beta$ & $1 \mathrm{~cm}$ \\
\hline
\end{tabular}

Table 2. Parameters used for segmentation of data sets 'Classroom' and 'Office room'.

\begin{tabular}{|c|c|c|c|c|c|c|c|c|}
\hline Scan & & $\boldsymbol{\Omega}(\mathbf{g o n})$ & $\mathbf{\Phi}(\mathbf{g o n})$ & $\mathbf{K}($ gon) & $\mathbf{T}_{\mathbf{x}}(\mathbf{m})$ & $\mathbf{T}_{\mathbf{y}}(\mathbf{m})$ & $\mathbf{T}_{\mathbf{z}}(\mathbf{m})$ & \#features \\
\hline \multirow{3}{*}{2} & Estimated value & 0.023499 & 0.111666 & 209.87202 & -2.198 & -2.134 & 0.002 & \multirow{2}{*}{14} \\
\cline { 2 - 8 } & Theor. Accuracy & 0.0019 & 0.0014 & 0.0018 & 0.00015 & 0.00021 & 0.00011 \\
\cline { 2 - 8 } & $\begin{array}{c}\text { Departure from } \\
\text { benchmarking result }\end{array}$ & 0.0037 & 0.0025 & 0.0021 & 0.00057 & 0.00032 & 0.00051 & \\
\hline
\end{tabular}

Table 3. Statistical results for 'Classroom' data set. 

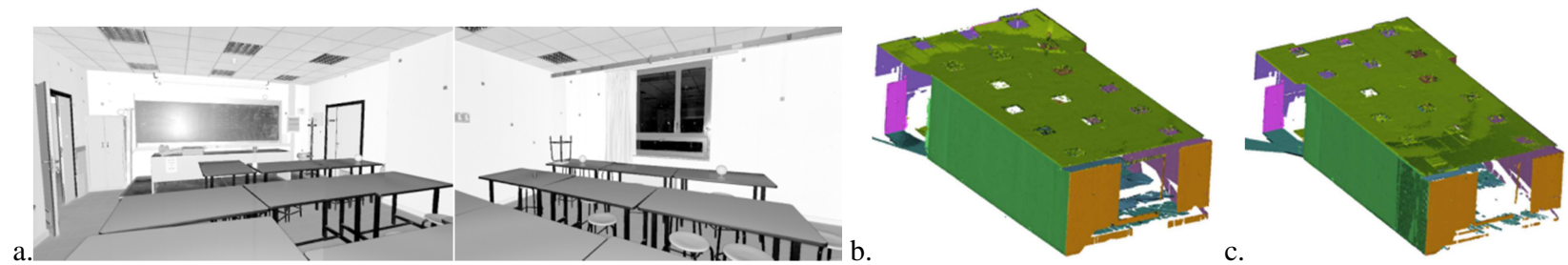

c.
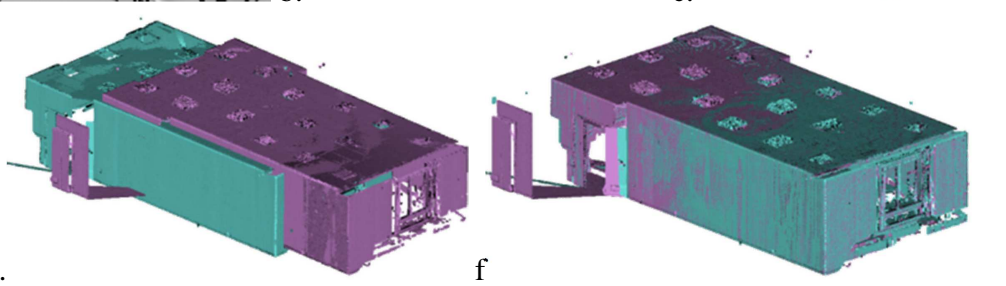

Figure 4. 'Classroom' data set registration results. Laser intensity images from bot scan stations (a); segmentation results for Scan 1 (b) and Scan 2 (c), where corresponding segments are represented with the same colour; estimated positions of scan stations (d); scan alignment before (e) and after the registration (f).

\section{2 'Office room' data set}

The second example consists in the contemporary registration of three scans of an office room (Fig. 5). The room is approximately $12 \mathrm{~m} \times 8.5 \mathrm{~m} \times 3 \mathrm{~m}$. Each scan consists of 44 million points resulting in average point density of about $1 \mathrm{pt} / 1 \mathrm{~mm}$. Also in this case the scans are acquired with FAROFOCUS 3D laser scanner. The parameters used for the segmentation of the scans are the ones presented in Table 2. The number of recovered objects in Scan 1, 2 and 3 is 20,18 and 22, respectively. Among these, 9 were matched across all scans, 15 between Scans 1 and 2, 13 between Scans 2 and 3, and 18 between Scans 1 and 3.
The coordinate system of Scan 1 was defined as GRS. The final 3D global adjustment of all scans was performed using point correspondences as described in the previous section. The final sigma naught was $3.2 \mathrm{~mm}$. Table 4 presents the standard deviations of registration parameters.

Also in this case a comparison to target-based registration was accomplished (see Tab. 4). Differences on artificial targets showed a mean difference of $2.4 \mathrm{~mm}$ and a standard deviation of $1.0 \mathrm{~mm}$ for Scan 2 and mean difference of $3.1 \mathrm{~mm}$ and a standard deviation of $0.9 \mathrm{~mm}$ for Scan 3, respectively.

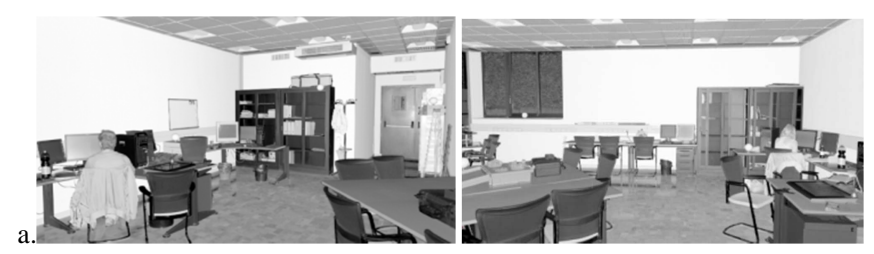

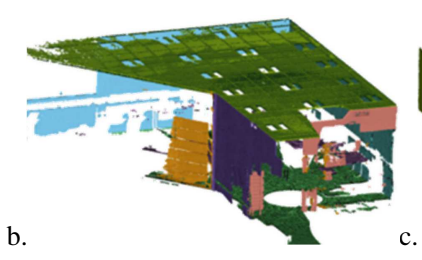

c.

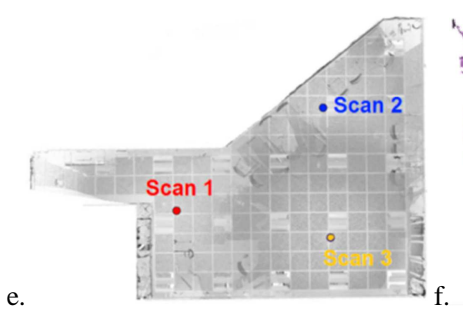

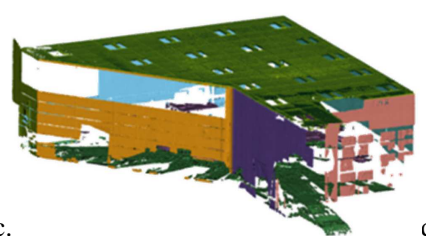

d.
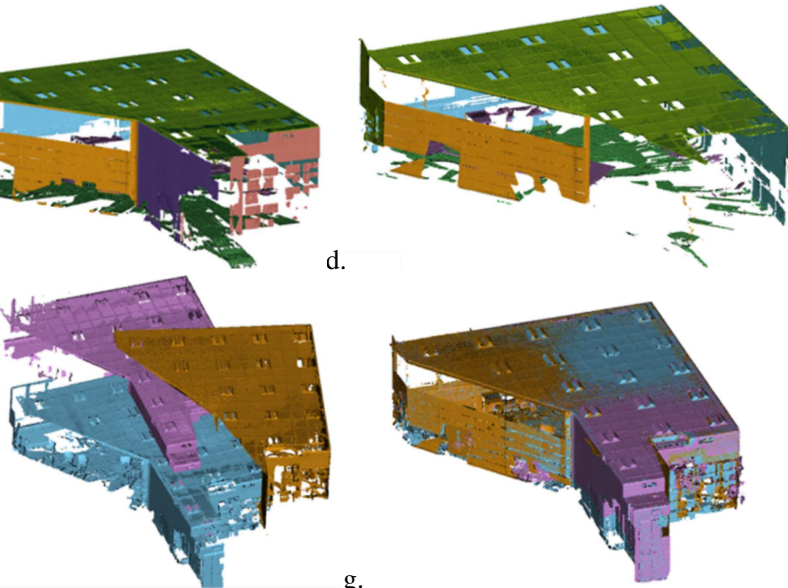

g.

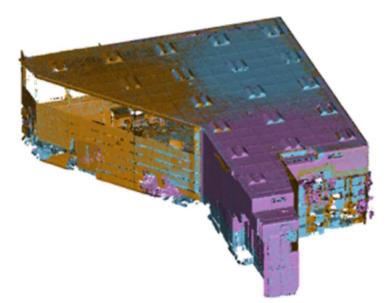

Figure 5. 'Office room' data set registration results. An overview of the office room (a); segmentation results for Scan 1 (b), Scan 2

(c) and Scan3(c), where corresponding segments are represented with the same colour; estimated positions of scan stations (e); and scan alignment before (f) and after the registration (g). 


\begin{tabular}{|c|c|c|c|c|c|c|c|c|c|}
\hline Scan & & $\Omega($ gon) & $\Phi$ (gon) & K (gon) & $\mathbf{T}_{\mathbf{x}}(\mathbf{m})$ & $\mathbf{T}_{\mathbf{y}}(\mathbf{m})$ & $\mathbf{T}_{\mathbf{z}}(\mathbf{m})$ & $\begin{array}{l}\text { \#features } \\
\text { in } 2 \text { scans }\end{array}$ & $\begin{array}{l}\text { \#features } \\
\text { in } 3 \text { scans }\end{array}$ \\
\hline \multirow[b]{3}{*}{2} & Estimated value & 0.25880 & 0.11833 & 292.07185 & -4.808 & 0.599 & 0.001 & \multirow[b]{3}{*}{18} & \multirow{3}{*}{ - } \\
\hline & Theor. Accuracy & 0.0019 & 0.0020 & 0.0019 & 0.00020 & 0.00015 & 0.00023 & & \\
\hline & $\begin{array}{c}\text { Departure from } \\
\text { benchmarking } \\
\text { result }\end{array}$ & 0.0028 & 0.0045 & 0.0038 & 0.00050 & 0.00041 & 0.00041 & & \\
\hline \multirow[b]{3}{*}{3} & Estimated value & -0.05979 & 0.33341 & 218.57519 & -0.427 & 3.992 & 0.002 & \multirow[b]{3}{*}{20} & \multirow[b]{3}{*}{9} \\
\hline & Theor. Accuracy & 0.0010 & 0.0018 & 0.0012 & 0.00027 & 0.00019 & 0.00013 & & \\
\hline & $\begin{array}{c}\text { Departure from } \\
\text { benchmarking } \\
\text { result }\end{array}$ & 0.0037 & 0.0041 & 0.0024 & 0.00048 & 0.00057 & 0.00048 & & \\
\hline
\end{tabular}

Table 4. Statistical results for 'Office room' data set.

\section{CONCLUSIONS}

This paper presented a method for registration of point clouds taken from different viewpoints on the basis of corresponding planar features. The developed technique makes use of an automatic segmentation algorithm that labels the points of a scan as belonging to a certain planar object. Once planar elements are extracted, correspondences between them are established. Registration parameters are then calculated by means of a non linear estimation process which is based on the minimization of the orthogonal distances of the plane parameters. An important aspect is the possibility of simultaneous registration of multiple point clouds, thus mitigating the accumulation of errors resulting from the concatenation of pairwise registrations. The presented method was applied to a couple of test data sets from an indoor university site, and the acquired scans were registered by using only the modelled objects. Additionally, quality estimates in terms of theoretical accuracy of scan and object parameters were computed.

Finally, we compared the presented approach to a target-based standard implementation. Results showed registration statistics comparable to the ones obtainable by using targets and with precision of the adopted instrument. This is mainly given by the fact that the indoor scenes typically present a high number of planar features with a good spatial distribution. Also their geometrical distribution was optimal for estimating in a reliable way the registration parameters. Indeed, planes were evenly distributed in all directions. Scenes with a lower number of planar features and a non-regular distribution of planes in space would give significantly worst results. This prevents a large and extensive use of the presented solution for registration of outdoor scenes. Indeed, in such case only few horizontal planes are expected, preventing a reliable estimation of shifts along the vertical direction.

\section{Acknowledgements}

Funding was partially provided by National Key Basic 973 Research Program of China (Id 2013CB733204).

\section{REFERENCES}

Acka D., 2007, Matching of 3D surfaces and their intensities. ISPRS Journal of Photogrammetry and Remote Sensing, 62(2): 112-121.

Alba M., Roncoroni F., Scaioni M., 2008, Investigations about the Accuracy of Target Measurement for Deformation Monitoring. International Archives of Photogrammetry, Remote Sensing and Spatial Information Sciences, 37 (Part B), 1053-1059.
Alba M.I., Barazzetti L., Scaioni M., Remondino F., 2011. Automatic Registration of Multiple Laser Scans using Panoramic RGB and Intensity Images. International Archives of Photogrammetry, Remote Sensing and Spatial Information Sciences, Vol. 38, (Part 5/12), 6 pp.

Al-Manasir K. and Fraser C.S., 2006, Registration of terrestrial laser scanner data using imagery. Photogrammetric Record, 21(115): 255268.

Awwad, T. M., Zhu, Q., Li, Q., 2009. An Extension of RANSAC. In Information Engineering and Computer Science, 2009. ICIECS 2009. International Conference on (pp. 1-4). IEEE.

Azhar S., 2011, Building information modeling (BIM): Trends, benefits, risks, and challenges for the AEC industry. Leadership and Management in Engineering 11, no. 3 (2011): 241-252.

Barnea S. and Filin S., 2010, Geometry-image-intensity combined features for registration of terrestrial laser scans. International Archives of Photogrammetry, Remote Sensing and Spatial Information Sciences, 38, (Part 3A), 145-149.

Bennamoun M. and Mamic G.J., 2002, Object Recognition: Fundamentals and Case Studies. Springer-Verlag, New York.

Besl P.J. and McKay N.D., 1992, A method for registration of 3-d shapes. IEEE Tans. Pattern Anal. Mach. Intell., 14: 239-256.

Böhm J., and Becker S. 2007, Automatic marker-free registration of terrestrial laser scans using reflectance features. In: Proc. $8^{\text {th }}$ Conf. on "Optical 3-D Measurement Techniques", Zurich, Switzerland, pp. 338344.

Boulaassal H, Landes T and Grussenmeyer P, 2008, Automatic extraction of planar clusters and their contours on building façades recorded by terrestrial laser scanner. VSMM 2008 - Conference on Virtual Systems and MultiMedia Dedicated to Digital Heritage. Limassol Cyprus - October 20th - 25th.

Boulaassal, H., Landes, T., Grussenmeyer, P., 2009. Automatic extraction of planar clusters and their contours on building façades recorded by terrestrial laser scanner. International Journal of Architectural Computing, 7(1), 1-20.

Dermanis A., 1994, The photogrammetric inner constraints. ISPRS Journal of Photogrammetry and Remote Sensing, 49(1), 25-39.

Dijkman S. and van den Heuvel F.A., 2000, Semi-automatic registration of laser scanner data. International Archives of Photogrammetry, Remote Sensing and Spatial Information Sciences 33 (Part B 5/1), 215221.

Dold C. and Brenner C., 2006, Registration of terrestrial laser scanning data using planar patches and image data. IAPRS, XXXVI, 5, 78-83.

Fischler M.A. and Bolles R.C., 1981, Random sample consensus: a paradigm for model fitting with applications to image analysis and automated cartography. Comm. ACM, 24(6), pp. 381-395.

Gellert W., Gottwald S., Hellwich M., Kästner H., Künstner H., 1989, VNR Concise Encyclopedia of Mathematics, 2nd ed. New York: Van Nostrand Reinhold, pp. 539-543, 1989.

Gruen A., 1985, Adaptive least squares correlation: a powerful image matching technique. South African Journal of Photogrammetry, Remote Sensing and Cartography, 14(3), 175-187. 
Gruen, A. and Akca D. 2005, Least squares 3D surface and curve matching. ISPRS Journal of Photogrammetry and Remote Sensing, 59(3), 151-174.

Guehring J., 2001, Reliable 3d surface acquisition, registration and validation using statistical error models. Proc.3rd International Conference on 3-D Digital Imaging and Modeling, Quebec, May 28June 1, pp. 224-231.

Kang Z., Li J., Zhang L., Zhao Q., Zlatanova S., 2009, Automatic registration of terrestrial laser scanning point clouds using panoramic reflectance images. Sensors, 9, pp. 2621-2646

Lichti D.D. and Gordon S.J., 2004, Error Propagation in Directly Georeferenced Terrestrial Laser Scanner Point Clouds for Cultural Heritage Recording. Proceeding of FIG Working Week, Athens, Greece, May 22-27, pp. 16.

Moussa, W. and D. Fritsch, 2014. Automatic Registration of NonOverlapping Laser Scans Based on a Combination Of Generated Images From Laser Data and Digital Images in One Bundle. Int. Arch. Photogramm. Remote Sens. Spatial Inf. Sci., 38-5/W12.

Novak, D. And K. Schindler, 2013. Approximate registration of point clouds with large scale differences. ISPRS Annals of the Photogrammetry, Remote Sensing and Spatial Information Sciences, Vol. II, Part 5/W2, pp. 211-216.

Rabbani, T., 2006. Automatic reconstruction of industrial installations using point clouds and images. Ph.D. thesis, Delft University of Technology, Delft, the Netherlands. 154 pages.

Rabbani T., Dijkman S., van den Heuvel F., Vosselman G., 2007, An integrated approach for modelling and global registration of point clouds. ISPRS journal of Photogrammetry and Remote Sensing, 61(6), 355-370.

Rusinkiewicz S. and Levoy M., 2001, Efficient variants of the ICP algorithm. Proc. of the 3rd International Conference on 3D Digital Imaging and Modeling, Quebec, May 28-June 1, pp. 145-152.

Sapkota, P.P., 2008. Segmentation of coloured point cloud data. M.Sc. thesis, ITC, Enschede, the Netherlands. 67 pages.

Scaioni M., 2005, Direct georeferencing of TLS in surveying of complex sites. International Archives of Photogrammetry, Remote Sensing and Spatial Information Sciences, 36, (Part 5/15), 8 pages.

Scaioni, M., 2012. On the estimation of rigid-body transformation for TLS registration. IAPRS\&SIS, Vol. XXXIX, Part B5, pp. 601-606

Scaioni M. and Forlani G., 2003, Independent model triangulation of terrestrial laser scanner data. International archives of photogrammetry remote sensing and spatial information sciences, 34, (5/12), 308-313.

Tóvári, D. and Pfeifer, N., 2005. Segmentation based robust interpolation-a new approach to laser data filtering. International Archives of Photogrammetry, Remote Sensing and Spatial Information Sciences, 36, (3/19): 79-84.

Van den Heuvel F.A., 1999, A line-photogrammetric mathematical model for the reconstruction of polyhedral objects. In: El-Hakim, S.F. (Ed.), Videometrics VI, Proceedings of International Society for Optical Engineering, vol. 3641, pp. 60-71.

Van Goor B., Lindenbergh R., and Soudarissanane S., 2011. Identifying corresponding segments from repeated scan data. Int. Arch. Photogramm. Remote Sens. Spatial Inf. Sci., XXXVIII-5/W12, 295300 .

Vosselman, G., 2004. Fusion of Laser Scanning Dara and Aerial Photographs for Building Reconstruction. In: International Archives of Photogrammetry and Remote Sensing, Vol. 34, (Part 3), Istanbul 2004.

Vosselman, G., Gorte, B. G. H., Sithole, G. and Rabbani, T., 2004. Recognising structure in laser scanner point clouds. International Archives of Photogrammetry, Remote Sensing and Spatial Information Sciences, 36, (Part8/W2): 33-38.

Vosselman G. and Maas H. G. (Eds.), 2010, Airborne and terrestrial laser scanning (Vol. 318). Dunbeath, UK: Whittles.

Wang Z. and Brenner C., 2008, Point based registration of terrestrial laser data using intensity and geometry features. In ISPRS Congress ('08), Beijing, China.

Wang Z., and Brenner C., 2008, Point based registration of terrestrial laser data using intensity and geometry features. In: IAPRSSIS, Beijing, China, Vol. XXXVII, Part B5, pp. 583-590.
Williams, J., and M. Bennamoun, 2001. Simultaneous registration of multiple corresponding point sets. CVIU, 81(1), pp. 117-142.

Zhang Z., 1994, Iterative point matching for registration of free-form curves and surfaces. International Journal of Computer Vision 13(2), $119-152$. 